\title{
STUDIES ON THE PHYTOCHEMICAL SCREENING AND FREE RADICAL SCAVENGING POTENTIALS OF SOLANUM NIGRUM LEAVES EXTRACT
}

\author{
SRIRAM PRASATH G ${ }^{1 *}$, ARCHANA PRAKASH ${ }^{1}$, SRINIVASAN PT ${ }^{1}$, SUBRAMANIAN S
}

${ }^{1} \mathrm{PG}$ and Research of Department of Biochemistry, Dwaraka Doss Goverdhan Doss Vaishnav College, Arumbakkam, Chennai, Tamil Nadu, India. ${ }^{2}$ Department of Biochemistry, University of Madras, Guindy Campus, Chennai, Tamil Nadu, India. Email: gsriramprasath@gmail.com

Received: 04 August 2016, Revised and Accepted: 15 August 2016

\section{ABSTRACT}

Objective: Solanum nigrum is paramount in medicinal perspective and belongs to family Solanaceae. From different parts of the plant, significant pharmacological and biological activities have been reported previously. This study was aimed to analyze the presence of various phytoconstituents and to determine the antioxidant potential, in vitro.

Methods: The ethanolic extract of leaves was investigated for phytochemical analysis, vitamin, and mineral content. The antioxidant (free radical scavenging) activity of the extract was determined against 2,2-diphenyl-1-picrylhydrazyl (DPPH) radical, 2,2'-azino-bis(3-ethylbenzothiazoline-6sulfonic acid) (ABTS), nitric oxide (NO), and superoxide scavenging assays.

Results: Phytochemical analysis of the leaves revealed the presence of phenols, alkaloids, flavonoids, glycosides, saponins, tannins, phytosterols, and triterpenoides. The leaves extract was found to contain appreciable amounts of flavonoids and phenols. The extract showed the presence of vitamins such as ascorbic acid, folic acid, and niacinamide. It has been found that the leaves of $S$. nigrum are rich in minerals such as copper, magnesium, manganese, vanadium, chromium, calcium, zinc, sodium, and potassium. S. nigrum leaves extract was found to be antioxidant in nature which is evident from DPPH, ABTS, NO, and superoxide radical scavenging assays.

Conclusion: The results of the present findings suggest that $S$. nigrum contains biologically important phytoconstituents, significant amounts of vitamins and minerals. In addition, S. nigrum leaves extract exert free radical scavenging potential in vitro.

Keywords: Medicinal plants, Solanum nigrum leaves, Phytoconstituents, Antioxidant.

(C) 2016 The Authors. Published by Innovare Academic Sciences Pvt Ltd. This is an open access article under the CC BY license (http://creativecommons. org/licenses/by/4. 0/) DOI: http://dx.doi.org/10.22159/ajpcr.2016.v9i6.14506

\section{INTRODUCTION}

Plants continue to be an important therapeutic aid for alleviating ailments of humankind. Search for eternal health, longevity of life, remedy to relieve pain and discomfort prompted, the early man to explore his immediate surroundings to search for therapeutic agents. Medicinal plants since times immemorial have been used in virtually all cultures as a rich source of medicine. The World Health Organization estimated that more than $80 \%$ of the population in developing countries relies on traditional medicine, mostly herbal medicines, for their primary health-care needs due to their availability, accessibility, and affordability. India is the largest producer of medicinal herbs and is called as "botanical garden of the world" [1].

Plants have the ability to synthesize a wide array of chemical compounds that protect them against the attack from a wide variety of predators such as microbes, insects, and herbivorous mammals. Some of these compounds, while being toxic to plant predators, turn out to have beneficial effects when used to treat human diseases. Such secondary metabolites are highly varied in structure; many are aromatic substances, most of which are phenols or their oxygen-substituted derivatives.

Natural products such as plants extract, either as pure compounds or as standardized extracts, provide unlimited opportunities for new drug discoveries because of the unmatched availability of chemical diversity. The therapeutic effects of herbs/herbal extract cannot be determined unless its active ingredient or cofactors are identified. Oneway to indicate strength is standardization to one or several marker compounds that are believed to be mainly responsible for the biological effects. Although phytotherapy continues to be used in several countries, most of the traditional medicinal plants have not received scientific or medical scrutiny. One such medicinal plant, which lacks scientific evidence for its wide folklore use, is S. nigrum.

\section{S. nigrum}

S. nigrum (European Black Nightshade or locally known as "black nightshade," Duscle, Garden Nightshade, Hound's Berry, Petty Morel, Wonder Berry, Small-fruited black nightshade or popolo) is a species in the Solanum genus which belongs to the family Solanaceae. The flowers have petals greenish to whitish, recurred when aged and surround prominent bright yellow anthers. In Tamil, $S$. nigrum is called as manathakkali. $S$. nigrum has been used traditionally to treat various ailments such as pain, inflammation fever, and enteric diseases [2-4]. It possesses many activities such as antitumorigenic, antioxidant [5], hepatoprotective [6], and diuretic [7]. The methanolic extract of berries of the plant S. nigrum possessed antioxidant and cardioprotective activity $[8,9]$. The ethanolic extract of $S$. nigrum was found to possess the antidiabetic property [10].

S. nigrum contains many active components are glycoalkaloids, glycoproteins, and polysaccharides, polyphenolic compounds such as gallic acid, catechin, protocatechuic acid, caffeic acid, epicatechin, rutin, and naringenin [11]. In this study, an attempt has been made to assess the nutritive value as well as the antioxidant potential of $S$. nigrum leaves extract.

\section{METHODS}

\section{Plant material}

The leaves of S. nigrum were collected from the local market in Chennai. The leaves were identified and authenticated by a taxonomist at the center for Advanced Studies in Botany, University of Madras.

\section{Preparation of extract}

The leaves of the plant were collected and washed thoroughly under running tap water and then were rinsed in distilled water; they were allowed to dry for some time. Then, these leaves $(2 \mathrm{~kg}$ ) were shadow dried without any contamination for about 3-4 weeks. 


\section{Delipidation and extraction}

The leaves of S. nigrum were dried at room temperature and powdered in an electrical grinder, which was then stored in an airtight container at $5^{\circ} \mathrm{C}$ until further use. The powdered leaves were delipidated with petroleum ether $\left(60-80^{\circ} \mathrm{C}\right)$ for overnight. Almost all the chlorophyll and lipid are deposited on the side of the flask and were removed carefully. The extraction was done with ethanol. The dried powder was subjected to Soxhlet using ethanol. The ethanolic extract was filtered, dried, and weighed. The extract obtained was evaporated in a rotary evaporator to get a powdery mass. The extract was dried under reduced pressure using rotator evaporator to get the crude. It was stored below $4^{\circ} \mathrm{C}$ until further use.

\section{Preparation of ash}

The $S$. nigrum leaves were shadow dried, finely powdered using the electrical grinder. $100 \mathrm{~g}$ of properly powdered seeds was taken in a vitreosil crucible and placed in an electrical muffle furnace overnight maintaining its temperature between $430^{\circ} \mathrm{C}$ and $450^{\circ} \mathrm{C}$ because the loss of zinc may occur at $>450^{\circ} \mathrm{C}$ and loss of potassium occur if the temperature is too high $\left(>480^{\circ} \mathrm{C}\right)$. The ash was then removed and dried in a vacuum desiccator. The yield of ash in the powdered leaves was found to be $6.03 \mathrm{~g} / 100 \mathrm{~g}$

\section{Trace element analysis}

About $2 \mathrm{~g}$ of ash was digested with a triple acid mixture comprising of nitric acid, sulfuric acid, and perchloric acid in the ratio of 11:6:3, respectively, for the complete removal of organic content. The digested sample was made up to $100 \mathrm{~mL}$ using deionized water, and this sample is used for the assay of trace elements through atomic absorption spectroscopy using hollow cathode lamps.

\section{Instrumentation and analytical procedures}

The determination of the trace element content of S. nigrum was carried out using an atomic absorption spectrometer (GBC-Avanta, Australia).

\section{Preliminary phytochemical screening}

The ethanolic extract of $S$. nigrum leaves was subjected to preliminary phytochemical screening of various plant constituents $[12,13]$.

\section{Determination of total phenolic content}

Total polyphenol content in the ethanol extract of $S$. nigrum was determined according to the Folin-Ciocalteu colorimetric method [14,15]. A standard curve was built with gallic acid reference solutions. Aliquots ranging from 2 to $10 \mathrm{~mL}$ of standard aqueous gallic acid solution $(100 \mu \mathrm{g} / \mathrm{mL})$ were pipetted into $100 \mathrm{~mL}$ volumetric flasks containing $70 \mathrm{~mL}$ of distilled water. Folin-Ciocalteu reagent $(5 \mathrm{~mL})$ and $10 \mathrm{~mL}$ of saturated sodium bicarbonate solution were added, and the volume was made up to $100 \mathrm{~mL}$ with distilled water. The solution was thoroughly mixed. The blank was prepared in the same manner but without gallic acid. After $1 \mathrm{hr}$ of incubation at room temperature, the absorbance was measured at $760 \mathrm{~nm}$. The samples were prepared in triplicates for each analysis, and the mean value was calculated. For the determination of the total phenolic content of $S$. nigrum leaves extract, aqueous solutions at the final concentration of $20 \mu \mathrm{g} / \mathrm{mL}$ were used; proceeding in the same manner described for the reference solutions, and the total polyphenolic content was expressed as $\mathrm{mg} / \mathrm{g}$ of gallic acid equivalents.

\section{Determination of total flavonoid content}

Total flavonoid content in the ethanolic extract of $S$. nigrum leaves was determined according to the method of Quettier-Deleu et al., 2000, with minor modifications [16]. A standard curve was built with quercetin reference solutions. Aliquots ranging from 2 to $8 \mathrm{~mL}$ of standard quercetin ethanol extract solution $(50 \mu \mathrm{g} / \mathrm{mL})$ were pipetted into $25 \mathrm{~mL}$ volumetric flasks containing $1 \mathrm{~mL}$ of $2 \%$ aluminum chloride dissolved in ethanol, and the volume was made up with ethanol. The blank was prepared by diluting $1 \mathrm{~mL}$ of $2 \%$ aluminum chloride dissolved in ethanol in a $25 \mathrm{~mL}$ volumetric flask with ethanol. After $1 \mathrm{hr}$ at room temperature, the absorbance was measured at $420 \mathrm{~nm}$. S. nigrum extract was evaluated at a final concentration of $20 \mu \mathrm{g} / \mathrm{mL}$, proceeding in the same manner described for the reference solutions and the total flavonoid content was calculated as quercetin equivalents (mg/g) from a calibration curve. The samples were prepared in triplicate for each analysis, and the mean value of absorbance was recorded.

\section{In vitro antioxidant assays}

2,2-diphenyl-1-picrylhydrazyl (DPPH) radical scavenging assay The free radical scavenging capacity of the ethanolic extract of $S$. nigrum was determined using DPPH [17]. DPPH $(200 \mu \mathrm{M})$ solution was prepared in $95 \%$ methanol. From the stock plant extract solution $200,400,800$, and $1000 \mu \mathrm{g} / \mathrm{mL}$ were taken in five test tubes. $0.5 \mathrm{~mL}$ of freshly prepared DPPH solution was incubated with test drug and after 10 minutes, absorbance was taken as $517 \mathrm{~nm}$ using a spectrophotometer. Standard ascorbic acid was used as a reference.

\section{2,2'-azino-bis(3-ethylbenzothiazoline-6-sulfonic acid) assay}

ABTS radical scavenging activity of ethanolic extract of $S$. nigrum was determined [18]. Briefly, ABTS radical cation $\left(\mathrm{ABTS}^{*}\right)$ was produced by mixing ABTS stock solution (7 mM in water) with 2.45 $\mathrm{mM}$ potassium persulfate and allowing the mixture to stand in the dark at room temperature for 12-16 hrs before use. Then, ABTS*+ solution was diluted with ethanol to an absorbance of 0.7 at $734 \mathrm{~nm}$. To $3.0 \mathrm{~mL}$ of diluted $\mathrm{ABTS}^{*+}$ solution, different concentrations (200, 400,800 , and $1000 \mu \mathrm{g} / \mathrm{mL}$ ) of leaves extract in ethanol were added and after 1 minute, the decrease in absorbance was measured at $734 \mathrm{~nm}$ spectrophotometrically.

\section{Assay for nitric oxide (NO) scavenging activity}

Sodium nitroprusside ( $5 \mathrm{mM}$ ) in phosphate buffer $\mathrm{pH} 7.7$ was incubated with $200,400,600$, and $800 \mu \mathrm{g} / \mathrm{mL}$ concentrations of drug dissolved in a suitable solvent (alcohol), and tubes were incubated at $25^{\circ} \mathrm{C}$ for 120 minutes. At intervals, $0.5 \mathrm{~mL}$ of incubation solution was removed and diluted with $0.5 \mathrm{~mL}$ of Griess reagent. The absorbance of the chromophore formed during diazotization of nitrite with sulfanilamide and subsequent $\mathrm{N}$-naphthyl ethylene diamine was measured at $546 \mathrm{~nm}$ [19].

\section{Superoxide anion (SO) radical scavenging assay}

The superoxide radical scavenging activity of S. nigrum was measured [20]. In this method, the activity is measured by reduction of riboflavin/light/ nitro blue tetrazolium (NBT). The $1 \mathrm{~mL}$ of reaction mixture contained phosphate buffer, NADH, NBT, and various concentrations $(200,400,800$ and $1000 \mu \mathrm{g} / \mathrm{mL}$ ) of the sample solution. The method is based on the generation of superoxide radical by auto-oxidation of riboflavin in the presence of light. The Superoxide radical reduces NBT to a blue-colored formazan that can be measured at $560 \mathrm{~nm}$.

\section{RESULTS}

The phytochemical analysis of $S$. nigrum leaves extract is presented in Table 1. The leaves extract is found to contain alkaloids, flavonoids, saponins, tannins, phytosterol, triterpenoids, glycosides, and Phenols.

The total phenolic and flavonoid contents were found to be $322 \pm 2.66 \mathrm{mg} / \mathrm{g}$ equivalents of gallic acid and $226 \pm 2.81 \mathrm{mg} / \mathrm{g}$ equivalents of quercetin, respectively.

Table 2 represents the mineral content of $S$. nigrum leaves extract. It has been found that the leaves of $S$. nigrum are rich in minerals such as copper, magnesium, manganese, vanadium, chromium, calcium, zinc, sodium, and potassium.

Figs. 1 and 2 represent the retention time for vitamin (standard) and the vitamin content of $S$. nigrum leaves. The leaves content contains ascorbic acid, folic acid, and niacinamide in appreciable amounts.

\section{In vitro antioxidant potential of $S$. nigrum leaves}

Figs. 3 and 4 show the dose-dependent effect of S. nigrum on the percentage inhibition of DPPH and ABTS radicals present in the reaction 
mixtures. The ethanolic extract of $S$. nigrum scavenges DPPH and ABTS radical in a concentration-dependent manner. The leaves extract of $S$. nigrum significantly and concentration-dependently reduced DPPH and ABTS radicals. However, at a concentration of $1000 \mu \mathrm{g} / \mathrm{mL}$, the extract significantly scavenged $84.45 \%$ of DPPH radicals and $85.45 \%$ ABTS radicals.

The NO and superoxide scavenging potential of the leaves extract are depicted in Figs. 5 and 6, respectively. The extract exhibited a maximum of $74.15 \%$ NO scavenging potential (Fig. 5) and $86.35 \%$ superoxide scavenging activity (Fig. 6).

\section{DISCUSSION}

Plants contain bioactive chemical substances that produce remarkable physiological and biochemical actions in the human body. These

Table 1: Phytochemical analysis of $S$. nigrum leaves extract

\begin{tabular}{ll}
\hline Phytoconstituents & Inference \\
\hline Alkaloids & + \\
Flavonoids & + \\
Saponins & + \\
Tannins & + \\
Phytosterol & + \\
Diterpenes & - \\
Triterpenoids & + \\
Glycosides & + \\
Anthraquinones & - \\
Phenols & + \\
\hline
\end{tabular}

Solanum nigrum: S. nigrum

Table 2: Mineral composition of $S$. nigrum leaves

\begin{tabular}{ll}
\hline Element & Concentration $(\boldsymbol{\mu g} / \mathbf{g})$ \\
\hline Calcium (Ca) & 210 \\
Magnesium (Mg) & 103 \\
Sodium (Na) & 110 \\
Potassium (K) & 50 \\
Zinc (Zn) & 10 \\
Iron (Fe) & 130 \\
Copper (Cu) & 2.3 \\
Vanadium (V) & 0.8 \\
Phosphorus (P) & 750 \\
\hline
\end{tabular}

Solanum nigrum: $S$. nigrum bioactive constituents include alkaloids, tannin, flavonoids, and phenolic compounds. Plant-derived natural products have received considerable attention in recent years due to diverse pharmacological properties including antioxidant and antitumor activity. Natural products/dietary phytochemical have aroused considerable interest in recent years as potential therapeutic agents to counteract free radical mediated diseases [21].

The most important of these bioactive compounds of plants are alkaloids, flavonoids, tannins, and phenolic compounds. Phytochemicals include compounds with various biological properties (i.e., antioxidant, antiproliferative, and DNA repair) which allow plants to cope up with environmental challenges including exposure to radiation and toxins [22]. They are bioactive compounds (secondary metabolites) found in plants that work with nutrients and dietary fibers to protect against diseases. Certain phytochemicals are almost structurally identical to insulin and act as an "insulin-like substances" that helps in the remedy of Type I and Type II diabetes. Most plants with antidiabetic properties have been found to contain secondary metabolites such as glycosides, alkaloids, and flavonoids [23]. It has been shown that many plants exhibit efficient antioxidant properties owing to their phenolic constituents. Earlier report indicated that phytochemical screening of this species indicates the presence of alkaloids, flavonols, flavones, flavanols, saponin, flavonoids, and steroids [24]. Alkaloids such as soladunalinidine, solasonine, and solamargine have been isolated from leaf of Solanum species [25].

The inorganic elements have been investigated as a potential preventive and treatment agents for both Type 1 and Type 2 diabetes mellitus. The differences in the concentration of these elements are attributed to the soil composition and climate, in which a plant grows. It has been found that the leaves of $S$. nigrum are rich in minerals such as copper, magnesium, vanadium, calcium, zinc, sodium, phosphorus and potassium which do play a pivotal role in insulin metabolism.

The leaves content contains ascorbic acid, folic acid, and niacinamide in appreciable amounts. Metal as micronutrient is important for the normal functioning of vital organs and is present in many enzymes which activate them, thereby influence the biochemical processes that are required in our diet.

The present study has shown that the $S$. nigrum leaves examined have an appreciable content of contain Ascorbic acid, folic acid, and niacinamide. The leaves contain minerals with an abundance of them in calcium, zinc, iron, manganese, and magnesium while they were least in

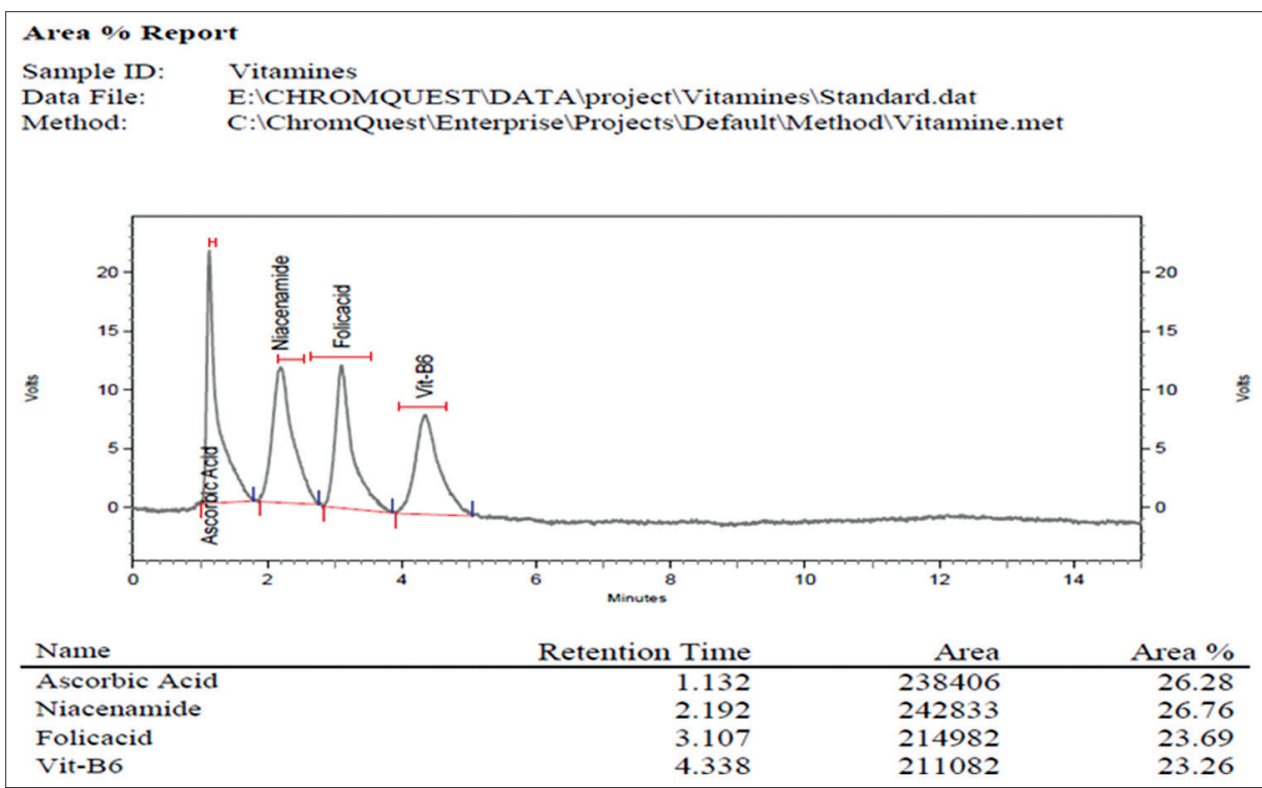

Fig. 1: Retention time for vitamins (standard) 


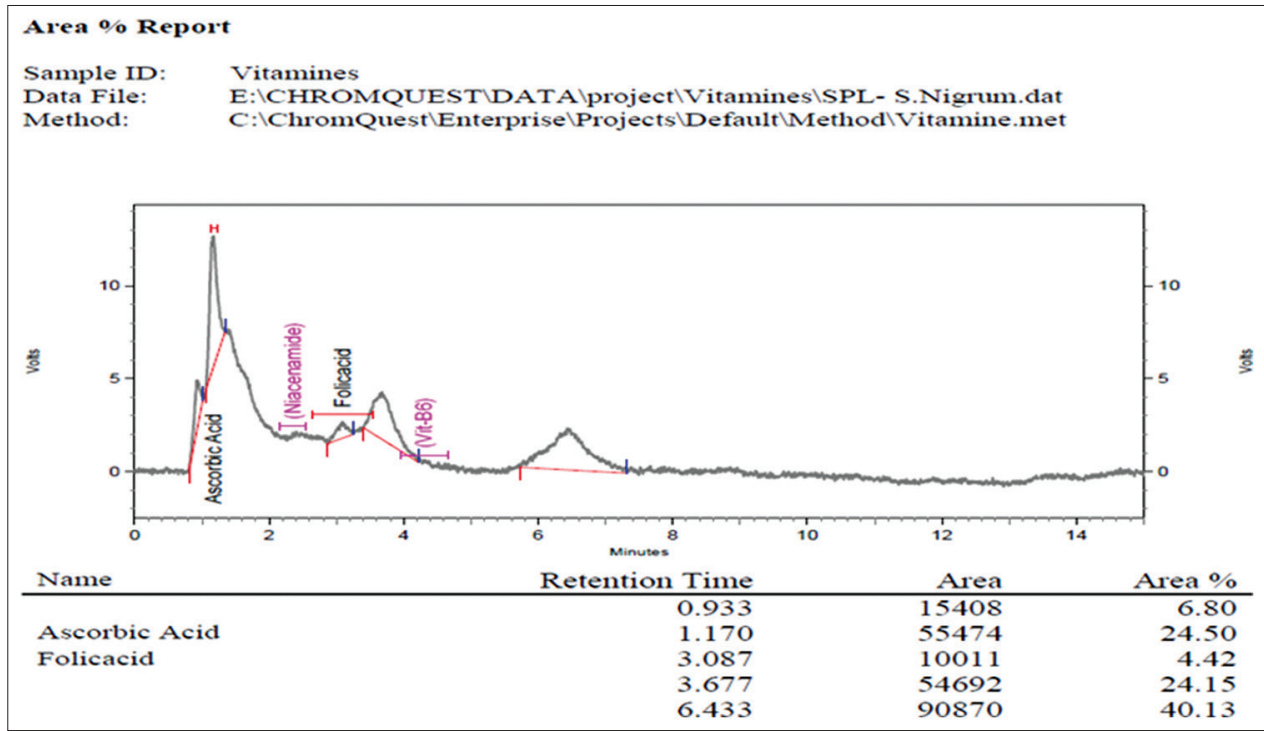

Fig. 2: Vitamin content of Solanum nigrum leaves extract

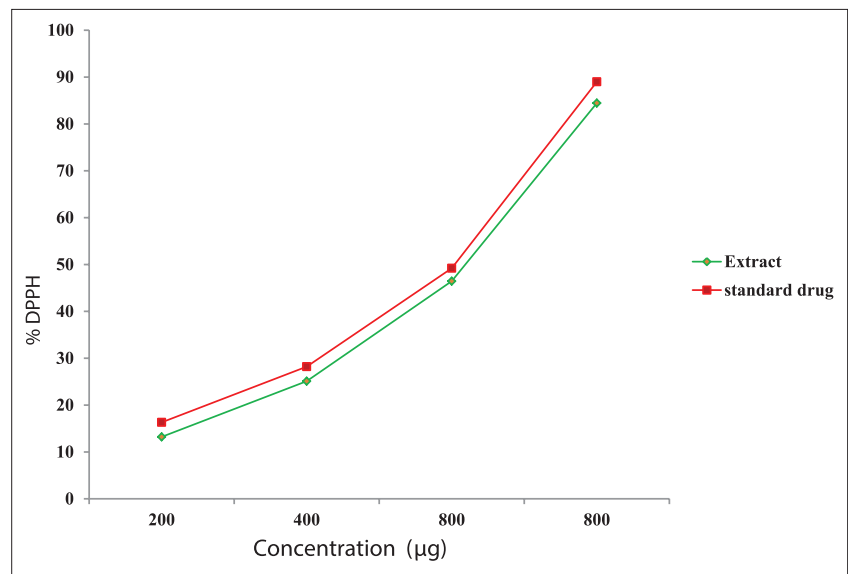

Fig. 3: 2,2-diphenyl-1-picrylhydrazyl radical scavenging potential of Solanum nigrum leaves extract

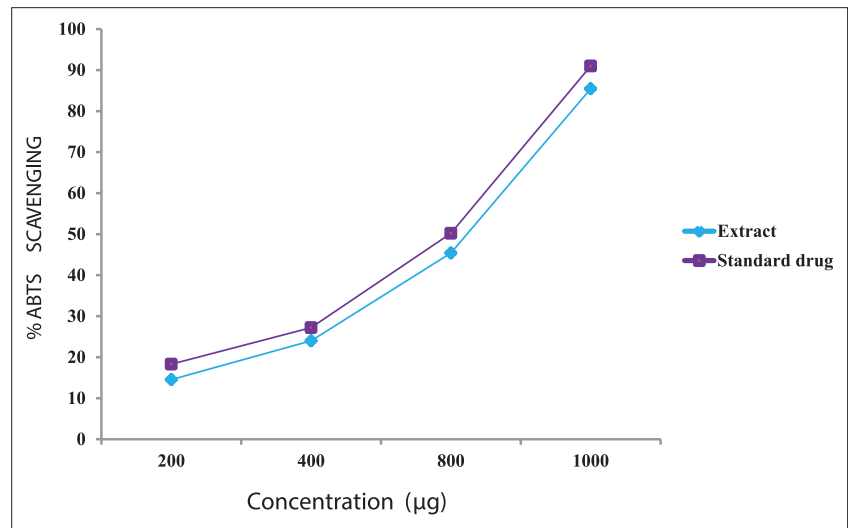

Fig. 4: 2,2'-azino-bis(3-ethylbenzothiazoline-6-sulfonic acid) radical scavenging potential of Solanum nigrum leaves extract

potassium. The results suggest that the leaves if consumed in sufficient amount would contribute greatly toward meeting human nutritional requirement for normal growth and adequate protection against free radical mediated diseases [26].

Phenolic compounds are known to be the most important antioxidants of plant materials. They contribute one of the major groups and

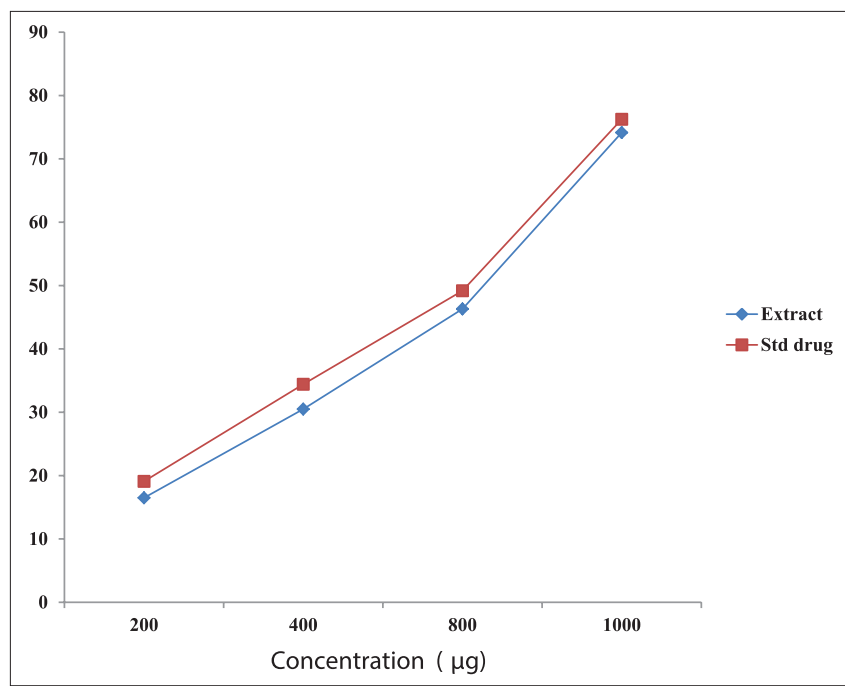

Fig. 5: Nitric oxide scavenging potential of Solanum nigrum leaves extract

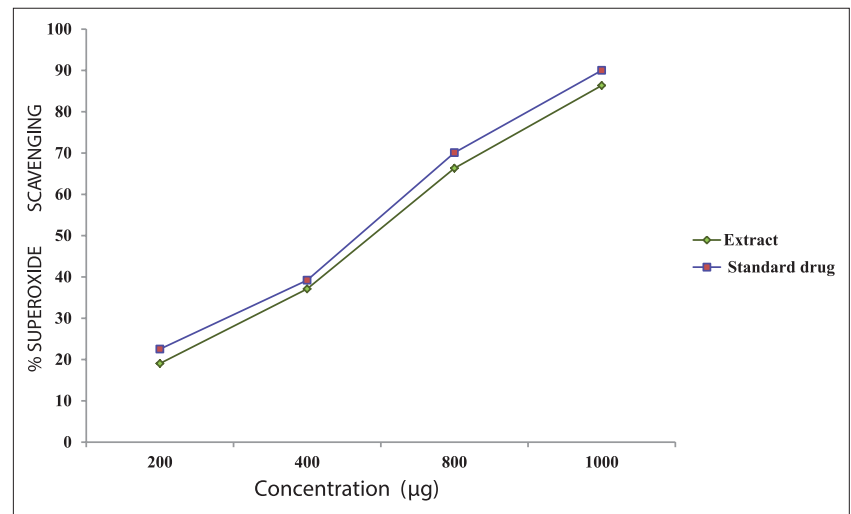

Fig. 6: Superoxide radical scavenging potential of Solanum nigrum leaves extract

compounds acting as primary antioxidants or free radical terminators. Antioxidant activity of a phenolic compound is based on their ability to donate hydrogen atoms to free radicals. In addition, they possess ideal structural properties for free radical scavenging properties. Flavonoids 
are a widespread group of natural compounds which possess a broad spectrum of chemical and biological activities including radical scavenging activity [27]. The presence of these compounds such as total phenolics and flavonoids in S. nigrum leaves extract may account for the antioxidant potential.

The free radical scavenging potential of natural products can be assessed by several assays. Among them, DPPH, ABTS, NO, and superoxide radical scavenging assays are routinely practiced for the assessment of antioxidant properties of different natural compounds, as they are easy, affordable, and reliable. In the present study, the antioxidant potential of $S$. nigrum leaves extract is examined by DPPH, ABTS, NO, and superoxide radical scavenging assays.

The ability of natural compounds to scavenge the DPPH radical can be expressed as its magnitude of antioxidative ability. DPPH radical in alcoholic solution is deep purple with an absorption peak at $515 \mathrm{~nm}$. DPPH assay is based on the principle that DPPH radical on accepting a hydrogen atom from the scavenger molecule, i.e., antioxidant, results in reduction of unpaired valence electron at one atom of nitrogen bridge in DPPH leading to the change of purple to yellow with concomitant decrease in absorbance at $515 \mathrm{~nm}$. The change in color from deep purple to yellow or the decrease in intensity signifies the antioxidant potential of the test compound.

ABTS assay is used for the screening of antioxidant activity of both water and lipid soluble compounds. The assay involves reduction of the color intensity of ethanolic solution containing pre-formed radical monocation of ABTS, generated by oxidation of ABTS with potassium persulfate due to the radical scavenging activity of antioxidants. The change in color intensity is proportional to the antioxidant efficiency of compounds.

The ethanolic extract of S. nigrum scavenges DPPH and ABTS radical in a concentration-dependent manner. The antioxidants react with DPPH, a purple-colored stable free radical, and convert it into a colorless $\alpha-\alpha$ diphenyl- $\beta$-picryl hydrazine. The amount of DPPH reduced could be quantified by measuring a decrease in absorbance at $517 \mathrm{~nm}$. ABTS assay is used for the screening of antioxidant activity of both water and lipid soluble compounds. The assay involves reduction of the color intensity of ethanolic solution containing pre-formed radical monocation of ABTS, generated by oxidation of ABTS with potassium persulfate due to the radical scavenging activity of antioxidants. The change in color intensity is proportional to the antioxidant efficiency of the compound. The leaves extract of $S$. nigrum significantly and concentration-dependently reduced DPPH and ABTS radicals. However, at a concentration of $1000 \mu \mathrm{g} / \mathrm{mL}$, the extract significantly scavenged $84.45 \%$ of DPPH radicals and $85.45 \%$ ABTS radicals.

The primary free radical in most biological systems is superoxide $\left(\mathrm{O}_{2}{ }^{--}\right)$. Although $\mathrm{O}_{2}^{-{ }^{--}}$itself is quite uncreative compared to the other radicals, it can be decomposed to form stronger oxidative species such as singlet oxygen and hydroxyl radicals. From the investigations, it was found that the $S$. nigrum leaf extract scavenged $\mathrm{O}_{2}{ }^{--}$significantly and in a concentration-dependent manner. The $\mathrm{O}_{2}^{-{ }^{-}}$scavenging activity was determined by phenazine methosulfate/NADH-NBT system wherein $\mathrm{O}_{2}{ }^{--}$derived from dissolved oxygen by phenazine methosulfate/NADH coupling reaction reduces NBT. The decrease of absorbance at $560 \mathrm{~nm}$ with antioxidants thus indicates the consumption of SOs in the reaction mixture. The leaves extract exhibited a maximum of $86.35 \%$ superoxide scavenging activity with a significant extent at a concentration of $1000 \mu \mathrm{g} / \mathrm{mL}$. S. nigrum leaf extract at a concentration of $1000 \mu \mathrm{g} / \mathrm{mL}$ also quenched $74.15 \%$ NO radical.

NO acts as neurotransmitter through exerting their effect on different body operations such as neurotransmission, synaptic plasticity, vasodilation, and central nervous system memory $[28,29]$. Besides the key role of $\mathrm{NO}$ in facilitating normal function, it has been observed that NO has been associated with pathophysiologic states such as neurodegenerative and Alzheimer's disease. Excessive release of NO in the body can cause DNA fragmentation, cell damage, and neuronal cell death $[30,31]$. Plants can play key role in reducing the amount of NO through their efficient NO scavenging activity.

S. nigrum exerts potent antioxidant activity. The antioxidants act as defense mechanism that protects against oxidative damage and includes compounds to remove or repair damaged molecules, and sufficient intake of antioxidants is supposed to protect against diseases. The phytochemical antioxidants have significant potential to neutralize free radicals or oxidants responsible for the cell damage. The present study thus scientifically validates and strengthens the candidature of $S$. nigrum in the preparation of medicinal aids to combat the wide spectrum of myriad diseases arising due to oxidative stress.

\section{CONCLUSION}

The results of the present study showed that S. nigrum leaves extract contains biologically active ingredients such as alkaloids, flavonoids, glycosides, saponins, triterpenoids, and phenols which possess a wide array of pharmacological properties. S. nigrum extract was found to contain appreciable amounts of vitamins and minerals. The leaves extract was found to be antioxidant in nature which is evident from $\mathrm{DPPH}, \mathrm{ABTS}, \mathrm{NO}$, and superoxide radical scavenging assays. These findings suggest that $S$. nigrum can be used in the treatment of free radical mediated diseases such as diabetes and cancer.

\section{ACKNOWLEDGMENT}

The authors wish to thank the management of DG Vaishnav College for having provided the facilities in the department to carry out the present study. The teaching and non teaching staffs of the Department of Biochemistry are gratefully acknowledged.

\section{REFERENCES}

1. Seth SD, Sharma B. Medicinal plants in India. Indian J Med Res 2004;120(1):9-11.

2. Rani P, Khullar N. Antimicrobial evaluation of some medicinal plants for their anti-enteric potential against multidrug resistant Salmonella typhi. Phytother Res 2004;18(8):670-3.

3. Zakaria ZA, Gopalan HK, Zainal H, Mohd Pojan NH, Morsid NA, Aris A, et al. Antinociceptive, anti-inflammatory and antipyretic effects of Solanum nigrum chloroform extract in animal models. Yakugaku Zasshi 2006;126(11):1171-8.

4. Acharya E, Pokhrel B. Ethno - Medicinal plants used by Bantar of Bhaudaha, Morang, Nepal. Our Nat 2006;4:96-103.

5. Lee SJ, Lim KT. Antioxidative effects of glycoprotein isolated from Solanum nigrum Linne on oxygen radicals and its cytotoxic effects on the MCF-7 cell. J Food Sci 2003;68:466-70.

6. Raju K, Anbuganapathi G, Gokulakrishnan V, Rajkapoor B, Jayakar B, Manian S. Effect of dried fruits of Solanum nigrum LINN against CCl4induced hepatic damage in rats. Biol Pharm Bull 2003;26(11):1618-9.

7. Li J, Li Q, Feng T, Zhang T, Li K, Zhao R, et al. Antitumor activity of crude polysaccharides isolated from Solanum nigrum Linne on U14 cervical carcinoma bearing mice. Phytother Res 2007;21(9):832-40.

8. Jainu M, Devi CS. Antioxidant effect of methanolic extract of Solanum nigrum berries on aspirin induced gastric mucosal injury. Indian J Clin Biochem 2004;19(1):57-61

9. Bhatia N, Maiti PP, Kumar A, Tuli A, Ara T, Khan MU. Evaluation of cardio protective activity of methanolic extract of Solanum nigrum Linn. in rats. Int J Drug Dev Res 2011;3(3):139-47.

10. Ali NS, Singh K, Khan MI, Rani S. Protective effect of ethanolic extracts of Solanum nigrum on the blood sugar of albino rats. Int J Pharm Sci Res 2010;1(9):97-9.

11. Ravi V, Saleem TS, Maiti PP, Gauthaman K, Ramamurthy J. Phytochemical and pharmacological evaluation of Solanum nigrum Linn. Afr J Pharm Pharmacol 2009;3(9):454-7.

12. Harborne JB. Methods of extraction and isolation. In: Phytochemical Methods. London: Chapman and Hall; 1998. p. 60-6.

13. Kokate CK. Practical Pharmacognosy. New Delhi, India: Vallabh Prakashan; 2005. p. 107-11.

14. Singleton VL, Orthofer R, Lamuela-Raventos RM. Analysis of total phenols and other oxidation substrates and antioxidants by means of Folin-Ciocalteu reagent. Method Enzymol 1999;299:152-78. 
15. Kumazawa S, Taniguchi M, Suzuki Y, Shimura M, Kwon MS, Nakayama T. Antioxidant activity of polyphenols in carob pods. J Agric Food Chem 2002;50(2):373-7.

16. Quettier-Deleu C, Gressier B, Vasseur J, Dine T, Brunet C, Luyckx M, et al. Phenolic compounds and antioxidant activities of buckwheat (Fagopyrum esculentum Moench) hulls and flour. J Ethnopharmacol 2000;72(1-2):35-42.

17. Brand-Williams W, Cuvelier ME, Berset C. Use of a free-radical method to evaluate antioxidant activity. Food Sci Technol Leb 1995;28:25-30.

18. Re R, Pellegrini N, Proteggente A, Pannala A, Yang M, Rice-Evans C. Antioxidant activity applying an improved ABTS radical cation decolorization assay. Free Radic Biol Med 1999;26:1231-7.

19. Marcocci L, Maguire JJ, Droy-Lefaix MT, Packer L. The nitric oxidescavenging properties of Ginkgo biloba extract EGb 761. Biochem Biophys Res Commun 1994;201(2):748-55.

20. Fontana M, Mosca L, Rosei MA. Interaction of enkephalins with oxyradicals. Biochem Pharmacol 2001;61:1253-7.

21. Devi S, Singh R. Antidiabetic activity of methanolic extract of Nepeta hindostana herb in streptozotocin induced diabetes in rats. Int J Pharm Pharm Sci 2016;8(7):330-5.

22. Huffman MA. Animal self-medication and ethno-medicine: Exploration and exploitation of the medicinal properties of plants. Proc Nutr Soc 2003;62(2):371-81
23. Craig W, Beck L. Phytochemicals: Health protective effects. Can J Diet Pract Res 1999;60(2):78-84.

24. Amir M, Kumar S. Possible industrial application of genus solanum in twenty first century - A review. J Sci Ind Res 2004;63:116-24.

25. Juneja D, Shrivastava PN, Guha MK, Saxena RC. Preliminary phytochemical screening of some folklore medicinal plants for their anti-inflammatory activity. Pharmacogn Mag 2007;3:11.

26. Kale MA, Bindu SM, Khadkikar P. Role of antioxidants and nutrition in oxidative stress: A review. Int J Appl Pharm 2015;7(1):1-4.

27. Ruba AA, Mohan VR. In vitro antioxidant potential of whole plant of Andrographis echioides (L.) nees (Acanthaceae). Int J Pharm Pharm Sci 2016;8(6):70-5.

28. Shibuki K, Okada D. Endogenous nitric oxide release required for long-term synaptic depression in the cerebellum. Nature 1991;349(6307):326-8

29. Bredt DS, Snyder SH. Nitric oxide: A physiologic messenger molecule. Annu Rev Biochem 1994;63:175-95.

30. Moncada S, Palmer RM, Higgs EA. Nitric oxide: Physiology, pathophysiology, and pharmacology. Pharmacol Rev 1991;43(2):109-42.

31. Dawson TM, Dawson VL, Snyder SH. A novel neuronal messenger molecule in brain: The free radical, nitric oxide. Ann Neurol 1992;32(3):297-311. 